\title{
Return to Work and Quality of Life after Stroke in Italy: A Study on the Efficacy of Technologically Assisted Neurorehabilitation
}

\author{
Sheyda Ghanbari Ghoshchi ${ }^{1,2,+}$, Sara De Angelis ${ }^{1,+}{ }^{,}$Giovanni Morone ${ }^{1}$, Monica Panigazzi ${ }^{3}$, \\ Benedetta Persechino ${ }^{4}$, Marco Tramontano ${ }^{1,5}{ }^{-}$, Edda Capodaglio ${ }^{3}$, Pierluigi Zoccolotti ${ }^{1,2}{ }^{\circ}$, \\ Stefano Paolucci ${ }^{1}$ and Marco Iosa ${ }^{1, *}$ \\ 1 IRCCS Fondazione Santa Lucia, Via Ardeatina 306, 00179 Rome, Italy; \\ sheyda.ghanbarighoshchi@uniroma1.it (S.G.G.); s.deangelis@hsantalucia.it (S.D.A.); \\ g.morone@hsantalucia.it (G.M.); m.tramontano@hsantalucia.it (M.T.); pierluigi.zoccolotti@uniroma1.it (P.Z.); \\ s.paolucci@hsantalucia.it (S.P.) \\ 2 Department of Psychology, Sapienza University of Rome, 00185 Rome, Italy \\ 3 Istituti Clinici Scientifici Maugeri IRCSS, Occupational Therapy and Ergonomics Unit, \\ 27040 Montescano, Italy; monica.panigazzi@icsmaugeri.it (M.P.); edda.capodaglio@icsmaugeri.it (E.C.) \\ 4 Italian Workers' Compensation Authority (INAIL), Department of Occupational and Environmental \\ Medicine, Epidemiology and Hygiene, Monte Porzio Catone, 00078 Rome, Italy; b.persechino@inail.it \\ 5 Department of Movement, Human and Health Sciences, University of Rome "Foro Italico", Interuniversity \\ Centre of Bioengineering of the Human Neuromusculoskeletal System, 00135 Rome, Italy \\ * Correspondence: m.iosa@hsantalucia.it \\ + These two authors contributed equally to this work.
}

Received: 5 June 2020; Accepted: 17 July 2020; Published: 20 July 2020

check for updates

\begin{abstract}
Cerebrovascular diseases, including stroke, are historically considered diseases of old adults so only in a few studies has "return to work" (RTW) been considered as an index of rehabilitative outcome. At the moment, data on RTW in patients with stroke are highly variable: four different reviews reported the following ranges: $11-85 \%, 19-73 \%, 22-53 \%$, and $40-45 \%$. The absence of re-integration to work after a stroke is shown to be associated with an increase of cardiac disorders and depression, with a higher level of mortality, with social isolation and with insufficient adaptive skills. The aim of this study was to verify the effectiveness of technological treatment, performed with optic (SonicHand) and wearable (Riablo ${ }^{\mathrm{TM}}$ ) systems providing auditory and visual biofeedback, on RTW in patients with stroke. RTW was found to be associated with a higher independence in the activities of daily living (assessed by the Modified Barthel Index). No significant differences were found between technological versus conventional rehabilitation in terms of RTW, despite the former showing a higher odds ratio than the latter ( $\mathrm{OR}=9$ vs. 6). Assistive devices were mainly used in patients who had not returned to work. Finally, quality of life was found higher in those patients who returned to work with the same conditions (work duties and time) as before stroke.
\end{abstract}

Keywords: stroke; wearable devices; return to work; rehabilitation; kinematics; quality of life

\section{Introduction}

Stroke is often considered a disease related to the elderly. In most studies, the rehabilitation outcome is the return to a proper level of independence in daily living activities. Few studies have used "return to work" (RTW) as an outcome index.

In the meantime, the mean age of stroke onset has decreased, with an increment of the incidence of stroke under 55 [1], and the retirement age in Italy has increased. The result is that the incidence of stroke 
in the working-age population has increased, and a higher number of people may be led to consider RTW as an important end-point of recovery after neurorehabilitation. The absence of re-integration at work after a stroke is shown to be associated with an increase of depression, social isolation, insufficient adaptive skills, and even with cardiac disorders and a higher level of mortality [2,3].

In Italy, the object of the present study, data on RTW and the recovery of social participation showed that only $19 \%$ of people after a stroke get back to work [4].

Return to work can be considered the highest point of the reintegration process, with positive consequences for the patient's health and well-being $[5,6]$. However, this stage is also very delicate, requiring individualized compliance with work activity in its organizational, structural and ergonomic aspects for this complex type of patient [5].

Hence, there is the need for greater accuracy in therapeutic choices and in controlling and rationalizing health care costs, and greater efficiency in achieving results optimized for return to work and quality of life, which requires a complex and very difficult approach [7].

Developments in technology-assisted rehabilitation have made possible the formulation of new rehabilitation paradigms derived from the integration of conventional rehabilitative programs with instrumental interventions specifically selected for the recovery of specific functions completely or partially lost with stroke [8,9]. These technologies may also allow professionals to have continuous and accurate data monitoring along with a summary of trends, that important for understanding and testing the effectiveness of treatments and the impact of observed variables $[10,11]$.

In fact, the rehabilitation project should also aim at recovering functional capacity as well as motor skills and social reintegration, along with RTW, key objectives in recovery after a disabling pathology such as stroke.

These new technologies for rehabilitation (i.e., instrumented biofeedback, serious exergames, virtual reality, collaborative robots [12]) may help patients with stroke to obtain a complete or, at least, a satisfying recovery of gross and fine motor functions, and also may favor individual autonomy regarding everyday life activities, including return to work.

Among these technologies, miniaturized and wireless wearable or optical sensors are increasingly being used for quantitative instrumental-based assessment of patients' functions and for their rehabilitation in neurological disorders [13-16].

Among these technologies, the sonification of hand and upper limb movements, using an optoelectronic device as biofeedback, has been recently proposed $[17,18]$, as have exergames for videogame-based therapy, controlled by wearable devices and a balance platform for the rehabilitation of postural and limb control and balance rehabilitation [19]. These technologically-assisted interventions may enhance the compliance of patients and, most importantly, act on neuronal mechanisms for re-learning through positive reinforcement [20].

The aim of this study is to assess return to work and the quality of life of patients with stroke after conventional rehabilitation and technological rehabilitation (performed with wearable and/or optic assistive devices), while identifying the prognostic factors for a satisfactory RTW.

\section{Materials and Methods}

\subsection{Trial Design}

The study design conformed to CONSORT Guidelines for a two-arm, single-blind randomized-controlled trial. The trial was approved by the Local Ethics Committee. All participants gave their written informed consent for participation in the study. A researcher who was not involved in the intervention sessions assessed the patients' eligibility to participate, based on the inclusion and exclusion criteria. 


\subsection{Participants}

This study was carried out in three Italian neurorehabilitation hospitals (IRCCS Fondazione Santa Lucia of Rome, IRCCS ICS Maugeri of Pavia and IRCCS ICS Maugeri of Montescano), where the enrolled patients had been hospitalized. In total, 50 patients with a diagnosis of stroke ( $>6$ months after stroke) were recruited according to the following inclusion criteria: subjects with a diagnosis of stroke occurring in working age (aged between 18 and 66 years) and with work activity at the time of the acute adverse event. Exclusion criteria included cognitive impairments within MMS (Mini-Mental State Examination) < 24, severe unilateral spatial neglect (assessed by the Letter Cancellation test, Barrage test, the Salford Sentence Reading Test, and the Wundt-Jastrow Area Illusion Test) and any severe comorbidities. Participants were randomly assigned to one of two groups; randomization was carried out by means of a computer-generated random numbers system. The two groups were the Technological Rehabilitation group (TG) and Control conventional rehabilitation Group (CG), as detailed in the following.

\subsection{Interventions}

All patients performed rehabilitation in the day-hospital regimen. Both groups performed the same number of rehabilitation sessions: two sessions of neuromotor rehabilitation per day and one session either of speech therapy, respiratory or phoniatric rehabilitation, three days per week for one month, each session lasting $40 \mathrm{~min}$. Both groups received the same amount of therapy. For TG, as detailed below, four hundred minutes of the above reported sessions of neuromotor rehabilitation were performed using technological devices. The CG performed all the above rehabilitation sessions according to conventional therapy, based on customized exercises focused either on hand dexterity and hand fine motor rehabilitation or on balance and postural improvement, according to prescribed physical exercises and rehabilitation targets, including the use of stabilization techniques and target achievements for the recovery of independence in the activities of daily living.

As stated above, patients allocated to the TG performed the same amount of therapy as the CG, but with the difference that four hundred minutes of the total hours of motor neurorehabilitation were performed using the SonicHand [21] or the Riablo ${ }^{\mathrm{TM}}$ [19], according to the individual established rehabilitation objectives defined by the clinical staff in accordance with the patient's expectancies. Both these devices provided technological biofeedback related to patients' movements. Riablo treatment was administered to patients with deficits related to postural balance and limb gross motor functions, but able to maintain upright posture at least for the time needed to perform the required tasks for the device [19]. SonicHand treatment was administered to patients with hand deficits and needing rehabilitation of upper limb fine motor functions and hand dexterity [17,21]. Despite some patients having both types of deficit, they received only one type from these two technologically assisted therapies. In these cases, two allocation criteria were followed: if the patients were confined toa wheelchair they were allocated to the SonicHand subgroup (because Riablo treatment cannot be administered to them); if patients were able to maintain the upright posture they received treatment according to personalized neurorehabilitation taking into account the most severe deficits (hand deficits vs. gross motor deficits) and the expectancies of patients and their families. The first of these two criteria led to SonicHand treatment being allocated to some of the most severely affected patients.

The SonicHand rehabilitation protocol [17] was in two phases. The first phase consisted of a 10 minutes warm-up including the exercises, complying with the individual needs and motor rehabilitation of each patient and chosen from wrist mobilization, opening and closing fingers and pronation and supination of the forearm. In the second phase which lasted for 10 minutes, at least six different exercises were proposed for the patient, choosing from: wrist radial and ulnar deviation, flexion and extension, pronation and supination; hand grasping, pinching and extension; shoulder and elbow flexion and extension and forward thrust. During these exercises, the movements were supported by the "sonification" technique. The sonification process was made using the Leap Motion Controller (LMC ${ }^{\circledR}$; Leap Motion, Inc., San Francisco, CA, USA), an optic assistive device, the software 
of which was adapted for rehabilitative purposes. The $L M C^{\circledR}$ is a human computer interface able to capture hand and finger movements and these data were transformed in sounds, thanks to a custom application developed with the Max 7 software programming environment (LMC ${ }^{\circledR}$; Leap Motion, Inc., San Francisco, CA, USA). The software program generated music sequences according to hand movements detected by the $\mathrm{LMC}^{\circledR}$. The application is able to associate movement with a four-note arpeggio or with a modulated texture. Based on the chosen exercises, sound parameters such as pitch, volume and spectrum have been identified and associated with the movement. The correct execution of the required movement allowed the production of a harmonic progression of arpeggios. The Sonic Hand protocol was performed for 20 sessions each lasting for 20 minutes.

The Riablo ${ }^{\mathrm{TM}}$ (CoRehab, Trento, Italy) is an adaptive system consisting of several wearable sensors and a stabilemetric platform wirelessly connected to software, to provide biofeedback through a video interface [19]. It allows the patients to enhance standard rehabilitation of balance and gross motor functions by guiding user's performances through the use of video-game based therapy. A protocol with six different exercises was proposed: the latero-lateral load shift; displacement of latero-lateral load on an oscillating platform; antero-posterior load shift; displacement of antero-posterior load on the oscillating platform; displacement of latero-lateral load with knee flexion; and lateral load displacement with knee flexion on an oscillating platform. All subjects performed 10 Riablo $^{\mathrm{TM}}$ training sessions, each lasting 40 minutes with an initial preparatory phase.

\subsection{Outcomes}

All patients were assessed with the Modified Barthel Index (MBI) [22], both at admission time (T0) and at discharge (T1). After almost six months after discharge, patients were contacted for a post-discharge telephone follow-up (FU).

During the phone call, all patients were assessed using the MBI [22,23] and the 12-Item Short Form Health Survey (SF-12) [24,25]. A specific questionnaire for RTW was also administered, together with questions related to any organizational or physical adaptations applied for the workplace. The Quebec User Evaluation of Satisfaction with Assistive Technology (QUEST) [26] and the Individual Prioritized Problems Assessment (IPPA) [27] were also administered but only to patients who used a specific assistive devices, prescribed by a physiatrist to patients during rehabilitation after stroke for increasing their independence in activities of daily living. These aids consisted of tripods, poles, wheelchairs or walkers. These were provided independently of belonging to the CG or TG and they did not refer to SonicHand or Riablo, but were provided to patients on the basis of physician assessment and of the detection of particular individual needs in order to cope with the autonomous execution of functional activities.

All the evaluation scores and the questionnaire data were collected by a researcher blinded to the allocation group.

\subsection{Statistical Analysis}

Binary data was reported using percentage values and analysed by $\chi^{2}$-test. Ordinal scores were reported using median and first and third quartiles and analysed using Mann-Whitney u-test. Continuous measures were reported using mean \pm standard deviations and analysed using a t-test. Forward binary logistic regression was used for identifying prognostic factors, and non-binary parameters were dichotomized using their median value. This last analysis allowed for computing the odds ratios (OR) and the relevant 95\% confidence intervals (CI95\%). For all the analyses the alpha level of statistical significance was set at $5 \%$.

\section{Results}

The final analysed sample consisted of 48 patients with stroke (two patients of TG were not traceable at follow-up), of whom 20 had returned to work (Figure 1). Data of the samples are reported in Table 1 for patients enrolled in TG and those in CG. The two groups were roughly balanced at 
admission, and no significant differences were found between them at further assessment. Table 1 also shows a comparison within TG of patients treated with Riablo $(N=10)$ and those with SonicHand $(N=13)$. Significant differences were found between these two subgroups for MBI-scores at the three assessment times and in the number of patients who experienced at least one fall after discharge, but not in terms of likelihood to return to work or quality of life.

Seeing as TG and CG were not significantly different, the data could be combined for the entire sample, as done in Table 2, in which comparisons were further performed between patients who returned to work and those who did not. Statistically significant differences were found between these patients in terms of MBI-scores at follow-up, physical score on SF-12 and number of patients needing an aid. At follow-up, 13 patients were using an assistive device. Table 3 reports features of patients who returned to work obtained by a specific questionnaire. Again, no significant differences were obtained between TG and CG patients. The MBI-scores at admission, discharge and follow-up are shown in Figure 2. No statistically significant differences were recorded between the two groups at each evaluation time.

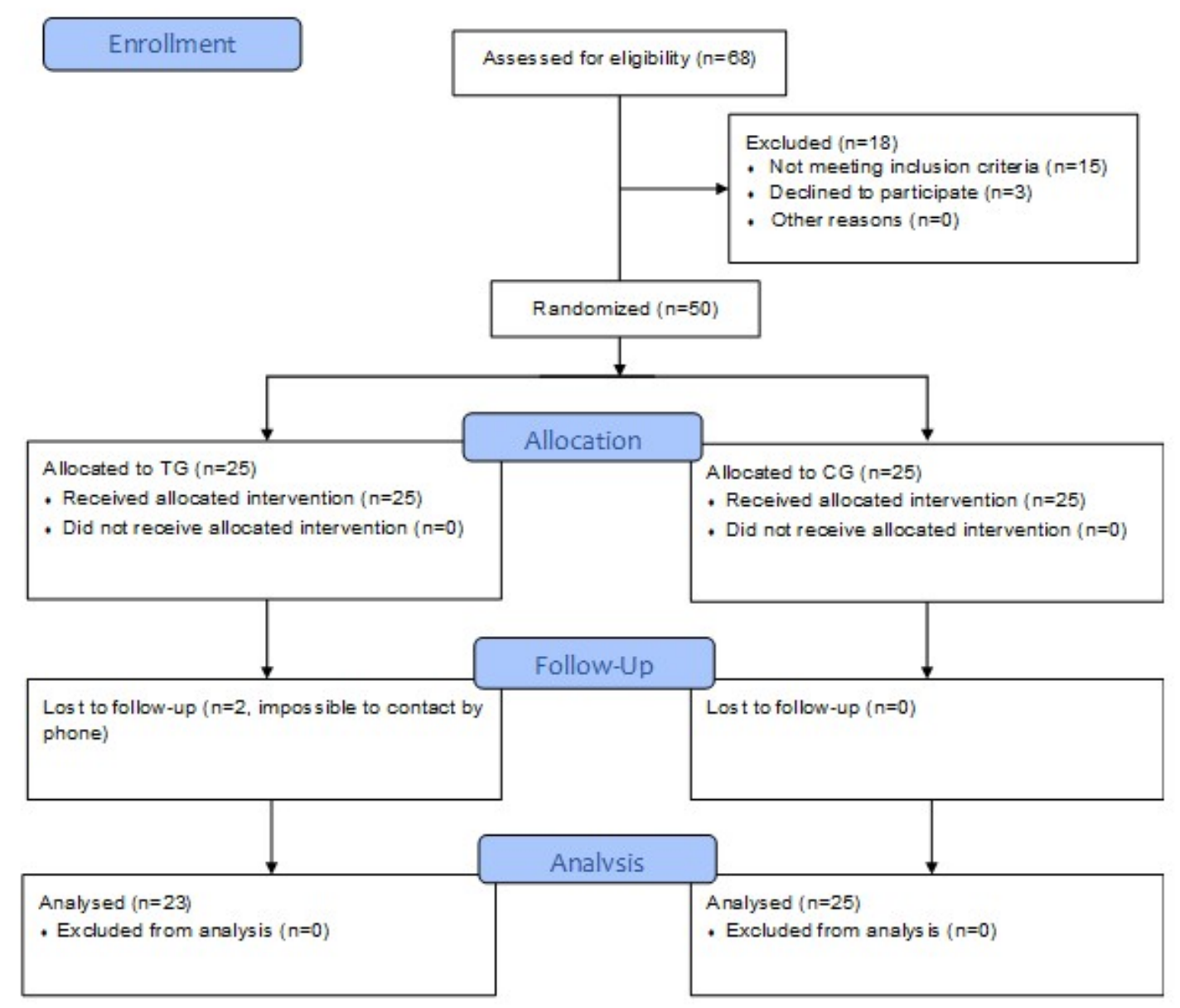

Figure 1. Flow Diagram. 


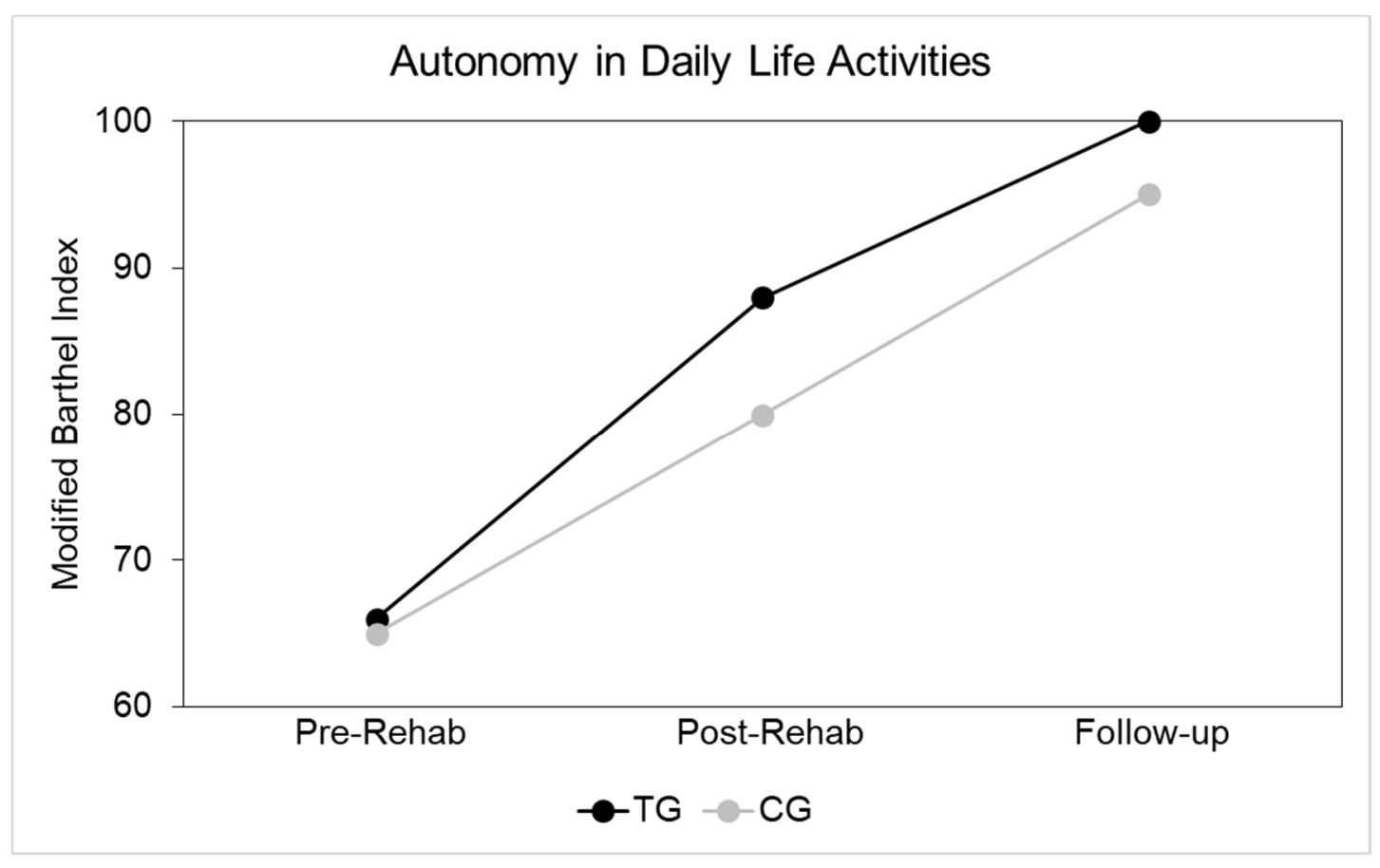

Figure 2. Median MBI-scores at admission, discharge and follow-up for control group (CG) (grey) and technological rehabilitation group (TG) (black).F.

Table 1. Patients (P.) data at the follow-up: absolute frequency (and percentages) for discrete variables, mean \pm standard deviation for continuous variables, median (and first and third quartiles) for ordinal data. The $p$-values (in bold if statistically significant) refer to the comparison between patients enrolled in technological group (TG) and those enrolled in the conventional therapy group (CG) in the fourth column and between patients who received Riablo versus SonicHand treatments in the sixth column. These p-values were obtained by the tests reported in the last column and selected according to the variable types.

\begin{tabular}{|c|c|c|c|c|c|c|c|}
\hline $\begin{array}{l}\text { Demographical and } \\
\text { Clinical Data }\end{array}$ & $\begin{array}{c}\text { TG } \\
(N=23)\end{array}$ & $\begin{array}{c}C G \\
(N=25)\end{array}$ & $p$-Value & $\begin{array}{l}\text { Riablo } \\
(N=10)\end{array}$ & $\begin{array}{l}\text { SonicHand } \\
(N=13)\end{array}$ & $p$-Value & Test \\
\hline Age (years) & $51.0 \pm 11.8$ & $52.5 \pm 10.5$ & 0.648 & $47.5 \pm 9.1$ & $54.0 \pm 13.3$ & 0.221 & t-test \\
\hline Months from stroke & $27.0 \pm 13.2$ & $21.7 \pm 12.2$ & 0.156 & $26.6 \pm 6.5$ & $27.0 \pm 16.9$ & 0.902 & t-test \\
\hline Male sex & $14(60.9 \%)$ & $17(68.0 \%)$ & 0.606 & $7(70 \%)$ & $7(53.8 \%)$ & 0.431 & $x^{2}$-test \\
\hline MBI Pre-Rehab & $66(41 ; 78)$ & $65(19 ; 75)$ & 0.793 & $79(74 ; 82)$ & $45(25 ; 60)$ & 0.002 & u-test \\
\hline MBI Post-Rehab & $88(72 ; 98)$ & $80(65 ; 90)$ & 0.391 & $98(95 ; 100)$ & $80(65 ; 87)$ & 0.005 & u-test \\
\hline MBI follow-up & $100(82 ; 100)$ & $95(85 ; 100)$ & 0.450 & $100(100 ; 100)$ & $90(65 ; 100)$ & 0.016 & u-test \\
\hline SF-12 total & $94(83 ; 106)$ & $97(84 ; 104)$ & 0.543 & $104(87 ; 109)$ & $92(75 ; 109)$ & 0.343 & u-test \\
\hline SF-12 physical & $47(39 ; 54)$ & $43(36 ; 49)$ & 0.190 & $48(45 ; 53)$ & $43(31 ; 54)$ & 0.563 & u-test \\
\hline SF-12 mental & $51(42 ; 58)$ & $58(48 ; 59)$ & 0.599 & $55(47 ; 59)$ & $50(38 ; 56)$ & 0.284 & u-test \\
\hline P. with assistive devices & $5(21.7 \%)$ & $8(32.0 \%)$ & 0.424 & $2(20 \%)$ & $3(23.1 \%)$ & 0.859 & $x^{2}$-test \\
\hline QUEST $1(N=13)$ & $4.7(4.4 ; 5.0)$ & $4.8(4 ; 4.8)$ & 0.602 & $4.7(4.6 ; 4.9)$ & $4.7(4.6 ; 4.9)$ & 0.767 & u-test \\
\hline QUEST $2(N=13)$ & $5(0.0 ; 5.0)$ & $2.5(0 ; 4.6)$ & 0.194 & $2.4(1.2 ; 3.6)$ & $5(5 ; 5)$ & 0.053 & u-test \\
\hline IPPA $1(N=13)$ & $20(19 ; 23.3)$ & $23(18.4 ; 25.0)$ & 0.303 & $19.5(19.2 ; 19.7)$ & $20(15 ; 21.6)$ & 0.767 & u-test \\
\hline IPPA $2(N=13)$ & $10(8 ; 10.5)$ & $15(10.5 ; 20)$ & 0.107 & $11.5(11.2 ; 11.7)$ & $7(6.8 ; 8.5)$ & 0.083 & u-test \\
\hline IPPA Difference $(N=13)$ & $8.0(5.0 ; 10)$ & $7.8(5 ; 10.2)$ & 0.605 & $8(8 ; 8)$ & $10(6.5 ; 13.3)$ & 0.554 & u-test \\
\hline P. with fall events & $5(21.7 \%)$ & $4(16.0 \%)$ & 0.611 & $0(0 \%)$ & $5(38.5 \%)$ & 0.026 & $x^{2}$-test \\
\hline Return to Work & $11(47.8 \%)$ & $9(36.0 \%)$ & 0.406 & $7(70 \%)$ & $4(30.8 \%)$ & 0.062 & $x^{2}$-test \\
\hline
\end{tabular}


Table 2. Patient data at follow-up: absolute frequency (and percentages) for discrete variables, mean \pm standard deviation for continuous variables, median (and first and third quartiles) for ordinal data. The $p$-values (in bold if statistically significant) refer to the comparison between subjects returned and not returned to work computed using the test reported in the last column according to the variable types.

\begin{tabular}{cccccc}
\hline $\begin{array}{c}\text { Demographical and Clinical } \\
\text { Data }\end{array}$ & Entire Sample & $\begin{array}{c}\text { Returned to } \\
\text { Work }\end{array}$ & $\begin{array}{c}\text { Not Returned } \\
\text { to Work }\end{array}$ & -Value & Test \\
\hline Number of subjects & 48 & $20(42 \%)$ & $28(58 \%)$ & 0.248 & $\chi^{2}$-test \\
\hline Age (years) & $51.8 \pm 11.1$ & $53.9 \pm 8.6$ & $50.3 \pm 12.5$ & 0.264 & t-test \\
\hline Time from stroke (months) & $24.2 \pm 12.8$ & $25.6 \pm 12.0$ & $23.2 \pm 13.5$ & 0.528 & t-test \\
\hline Male sex & $31(64.6 \%)$ & $13(65.0 \%)$ & $18(64.3 \%)$ & 0.959 & $\chi^{2}$-test \\
\hline MBI follow-up & $100(91 ; 100)$ & $100(100 ; 100)$ & $100(70 ; 100)$ & $\mathbf{0 . 0 1 8}$ & u-test \\
\hline SF-12 total & $96(83 ; 107)$ & $100(87 ; 109)$ & $93(77 ; 105)$ & 0.143 & u-test \\
\hline SF-12 physical & $45(36 ; 51)$ & $48(44 ; 55)$ & $43(33 ; 48)$ & $\mathbf{0 . 0 0 9}$ & u-test \\
\hline SF-12 mental & $52(42 ; 59)$ & $53(41 ; 59)$ & $52(43 ; 58)$ & 0.983 & u-test \\
\hline Patients with assistive devices & $13(27.1 \%)$ & $1(5.0 \%)$ & $12(42.8 \%)$ & $\mathbf{0 . 0 0 4}$ & $\chi^{2}$-test \\
\hline QUEST 1 $(\mathrm{N}=13)$ & $4.7(4.1 ; 5.0)$ & 4.8 & $4.7(4.4 ; 5)$ & 0.683 & u-test \\
\hline QUEST 2 $(\mathrm{N}=13)$ & $4.5(0.0 ; 5.0)$ & 0 & $4.6(0.7 ; 5)$ & 0.209 & u-test \\
\hline IPPA 1 $(\mathrm{N}=13)$ & $20(17.8 ; 24.1)$ & 25 & $20(18.4 ; 23.3)$ & 0.227 & u-test \\
\hline IPPA 2 (N = 13) & $11.3(7.5 ; 18.9)$ & 20 & $11.2(7.7 ; 13.7)$ & 0.228 & u-test \\
\hline IPPA Difference (N = 13) & $8.0(5.0 ; 10.5)$ & 5 & $8.0(7.0 ; 10.3)$ & 0.346 & u-test \\
\hline Patients with fall events & $9(18.7 \%)$ & $2(10.0 \%)$ & $7(25.0 \%)$ & 0.189 & $\chi^{2}$-test \\
\hline Technological treatment & $23(47.9 \%)$ & $11(55.0 \%)$ & $12(42.9 \%)$ & 0.406 & $\chi^{2}$-test \\
\hline & & & &
\end{tabular}

Table 3. Data of patients returned to work and comparison of TG and CG.

\begin{tabular}{cccccc}
\hline $\begin{array}{c}\text { Return to Work } \\
\text { Data }\end{array}$ & $\begin{array}{c}\text { Entire Sample of } \\
\text { Returned to Work }\end{array}$ & $\begin{array}{c}\text { Technological } \\
\text { Treatment } \\
\text { Group }\end{array}$ & $\begin{array}{c}\text { Conventional } \\
\text { Treatment } \\
\text { Group }\end{array}$ & $p$-Value & Test \\
\hline Number of subjects & 20 & 11 & 9 & 0.655 & $\chi^{2}$-test \\
\hline Working Hours & $32.6 \pm 9.3$ & $33.7 \pm 9.0$ & $31.1 \pm 10.1$ & 0.552 & $\chi^{2}$-test \\
\hline $\begin{array}{c}\text { People with any } \\
\text { kind of adaptation }\end{array}$ & $9(45.0 \%)$ & $4(36.4 \%)$ & $5(55.5 \%)$ & 0.888 & $\chi^{2}$-test \\
\hline $\begin{array}{c}\text { People with job } \\
\text { adaptation }\end{array}$ & $4(20.0 \%)$ & $1(9.1 \%)$ & $3(33.3 \%)$ & 0.391 & $\chi^{2}$-test \\
\hline $\begin{array}{c}\text { People with time } \\
\text { adaptation }\end{array}$ & $7(35.0 \%)$ & $4(36.4 \%)$ & $3(33.3 \%)$ & 0.178 & $\chi^{2}$-test \\
\hline $\begin{array}{c}\text { People with tools } \\
\text { adaptation }\end{array}$ & $0(0 \%)$ & $0(0 \%)$ & $0(0 \%)$ & - & - \\
\hline $\begin{array}{c}\text { People with } \\
\text { assistive devices }\end{array}$ & $1(5.0 \%)$ & $0(0 \%)$ & $1(11.1 \%)$ & 0.257 & $\chi^{2}$-test \\
\hline
\end{tabular}

Table 4 shows the results of the two Forward Binary Logistic Regressions. The first regression was performed using return to work as the main outcome, and hence as a dependent variable. The only factor found to be statistically influencing RTW was the MBI score at follow-up, with a probability of 7.5 times higher for patients who had an MBI equal or higher than 95 to return to work compared to other patients. A further analysis was conducted, separating TG and CG on the possibilities of returning to work, showing an Odds Ratio OR $=9(p=0.027)$ and $\mathrm{OR}=6(p=0.05)$, respectively. 
The second regression analysis showed that a good quality of life, assessed by an SF-12 total score higher than 95, was influenced by RTW on the same conditions as before stroke (OR $=6.6, p=0.026$ ), and neither just by RTW ( $p=0.613$ ), nor by a good level of autonomy (MBI f-up: $p=0.383$ ). Eleven subjects returned to work on the same conditions, same work duties and same work times as before the stroke event.

Table 4. Results of Forward Binary Logistic Regression (OR: odds ratio; CI95\%: 95\% confidence interval, $\mathrm{p}$-Value in bold if statistically significant).

\begin{tabular}{|c|c|c|c|c|c|c|}
\hline $\begin{array}{l}\text { Dependent } \\
\text { Variable }\end{array}$ & $\begin{array}{c}\text { Variables into } \\
\text { the Model }\end{array}$ & OR & $p$-Value & CI95\% & $\begin{array}{l}\text { Explained } \\
\text { Variance }\end{array}$ & Variables out of the Model \\
\hline $\begin{array}{l}\text { Return to } \\
\text { work }\end{array}$ & MBI f-up & 7.5 & 0.002 & $\begin{array}{c}2.04 \\
27.59\end{array}$ & $72.9 \%$ & $\begin{array}{c}\text { Age: } p=0.527 \\
\text { Gender: } p=0.805 \\
\text { Treatment: } p=0.728 \\
\text { Time from stroke: } p=0.610 \\
\text { MBI pre-R: }=0.128 \\
\text { MBI post- } \mathrm{R}=0.740\end{array}$ \\
\hline $\begin{array}{c}\text { SF-12 } \\
\text { Total score }\end{array}$ & $\begin{array}{c}\text { Work as } \\
\text { before (job } \\
\text { type and time) }\end{array}$ & 6.6 & 0.026 & $1.2 ; 34.9$ & $64.6 \%$ & $\begin{array}{c}\text { Age: } p=0.459 \\
\text { Treatment: } p=0.449 \\
\text { Time from stroke: } p=0.174 \\
\text { Gender: } p=0.497 \\
\text { Return to work: } p=0.613 \\
\text { MBI pre-R: } p=0.331 \\
\text { MBI post-R: } p=0.269 \\
\text { MBI f-up: } p=0.383\end{array}$ \\
\hline
\end{tabular}

\section{Discussion}

Participation in the work role is important as it enhances social reintegration and may influence quality of life, but the percentage of patients returning to work after stroke is still low. In this study, RTW occurred in $42 \%$ of subjects. These results are in line with previously reported data of $40-45 \%$ RTW [4,28,29]. Other studies have reported higher variability for RTW: 11-85\% [28], 19-73\% [4], or $22-53 \%$ [29]. In our specific study, the features of patients returned to work are those reported in Table 3: they were more independent in the activities of daily living (MBI at follow-up), with a higher quality of life in the physical domain, and fewer needed an assistive device when compared to those not returned to work. These results were summarized by logistic regression analysis in the identification by Modified Barthel Index assessed at follow-up as the only consistent prognostic factor related to RTW. In our study, age did not show a statistically significant relation to RTW. However, after a stroke, one of the main determinants of returning to work seems to be working age [28,30-32]. Barker-Collo et al. [33] showed that age was an important predictor of RTW. The discrepancy with our results may be due to the fact that age may have indirectly influenced RTW by affecting the level of independence in the activities of daily living, a factor directly assessed in our study by means of MBI-fup. Despite this parameter being slightly higher in the technologically treated group, the effect of this type of treatment was not significantly higher than that of conventional treatment. These results did not support previous studies reporting that new modern technological interventions have a higher positive impact on stroke rehabilitation than conventional interventions [34,35]. Patients allocated to the technological group could be treated with SonicHand if their main deficit was related to the hand, or with Riablo if they had postural and limb gross motor deficits. Severe patients confined to wheelchair could perform the SonicHand treatment, but not Riablo, because this last device needs an upright posture for performing balance and limb motor training. It was expected that the technological subgroup treated with SonicHand would be more problematic than that treated with Riablo, showing significantly lower scores of MBI in all three assessment times. The different severity and also the specific effects of Riablo treatment, on balance [19], may explain the reasons for which none of the patients treated with Riablo experienced a fall after discharge, whereas $38 \%$ of patients treated with SonicHand (and $25 \%$ of CG) 
fell at least once after discharge. Despite these differences, neither likelihood of return to work nor quality of life was significantly different between the two technological subgroups.

We found only an indirect effect of technological rehabilitation: a significant higher odds ratio was found in favor of technological rehabilitation versus conventional in achieving a higher MBI-score at follow-up; this latter parameter was then found to be directly influencing RTW. It seems that, with the same autonomy achieved, patients treated with technological rehabilitation are more likely to return to work than those treated with conventional rehabilitation. At the same time, conventional therapy has shown the advantage of also allowing some less independent patients to return to work. These findings are in line with previous studies showing that technological rehabilitation could be more effective for specific patients (depending on the degree of severity with respect to the type of technology), whereas conventional therapy could have a generalized positive effect [36-38].

One of the most interesting results of our study was that return to work by itself was not sufficient to warrant good quality of life. Quality of life was higher in subjects who returned to work without any losses in terms of time or working duties. To achieve this, a highly specialized rehabilitation is needed, and this should be integrated with specific interventions in the workplace. Conversely, in our study, only one patient of those who returned to work received an assistive device, showing that there is the need to improve the culture of RTW after stroke, satisfying the needs of subjects for a complete recovery of job status similar to that enjoyed before the stroke. In fact, only subjects who returned to the same job and at the same working conditions as before stroke reported a high quality of life.

Our study has some limitations. The dimension of the workplace and specific characteristics of the job were not investigated, as well as social characteristics of the job. Despite the fact that we recorded this information during phone follow-up, the sample size of our study limited the significance of the comparisons between the different technologies used in TG and did not allow us to divide subjects in subgroups related to the type of their job. The economic impact on the family of patients relating to RTW was not assessed in this study, but this could be a fundamental aspect leading the decision not to return to work in patients with moderate independence in the activities of daily living. Finally, psychological aspects should be investigated in further studies to analyze the factors influencing RTW and those influenced by RTW, together with other possible factors, such as pain [39] and patients' needs [40]. Along these lines, psychosocial support should be considered as a critical aspect of stroke rehabilitation. Technological rehabilitation could be helpful for people who use technological devices in their work but may be less useful in subjects not used to interacting with modern technologies [41-43].

In conclusion, our study has shown that quality of life after stroke is strongly influenced by the possibility of returning to work under the same conditions as before the acute event. This may depend on the independence achieved by patients, which was not superior under one type of rehabilitation versus the other type. Furthermore, patients enrolled in our study achieved a good level of independence, highlighting the importance of personalized rehabilitative treatments focused on the characteristics and the needs of each patient. Finally, our study recommends institutions to favor the development of facilities in workplaces that can accommodate patients and help them to adapt to their job, without the constraint of reduced working duties or working time.

Author Contributions: Conceptualization, M.P. and M.I.; Methodology, M.P., M.I., G.M. and M.T.; Formal Analysis, M.I.; Investigation, S.G.G., S.D.A. and E.C.; Resources, B.P.; Data Curation, M.I.; Writing-Original Draft Preparation, S.G.G. and S.D.A; Writing-Review \& Editing, P.Z., S.P. and E.C.; Supervision, P.Z. and S.P.; Project Administration, M.P., M.I. and B.P.; Funding Acquisition, M.P., M.I. and B.P. All authors have read and agreed to the published version of the manuscript.

Funding: This work was supported by the Italian National Institute for Insurance against Accidents at Work (INAIL), in the framework of BRIC project: "PROGETTO STAR: Innovative strategies, and approaches for the motor and functional rehabilitation of subjects with neurovascular adverse event outcomes for reintegration into work".

Conflicts of Interest: The Authors declare that there is no conflict of interest.

Data Availability Statement: The data that support the findings of this study are available from the corresponding author [MI] upon reasonable request. 


\section{References}

1. Boehme, A.K.; Esenwa, C.; Elkind, M.S. Stroke Risk Factors, Genetics, and Prevention. Circ. Res. 2017, 120, 472-495. [CrossRef] [PubMed]

2. Gallo, W.T.; Bradley, E.H.; Teng, H.M.; Kasl, S.V. The effect of recurrent involuntary job loss on the depressive symptoms of older US workers. Int. Arch. Occup. Environ. Health 2006, 80, 109-116. [CrossRef]

3. Edwards, J.D.; Kapoor, A.; Linkewich, E.; Swartz, R.H. Return to work after young stroke: A systematic review. Int. J. Stroke 2018, 13, 243-256. [CrossRef] [PubMed]

4. Treger, I.; Shames, J.; Giaquinto, S.; Ring, H. Return to work in stroke patients. Disabil. Rehabi. 2007, 29, 1397-1403. [CrossRef]

5. Wolfenden, B.; Grace, M. Returning to work after stroke: A review. Int. J. Rehabil. Res. 2009, 32, 93-97. [CrossRef] [PubMed]

6. Koch, L.; Egbert, N.; Coeling, H.; Ayers, D. Returning to Work after the Onset of Illness: Experiences of Right Hemisphere Stroke Survivors. Rehabil. Couns. Bull. 2005, 48, 209-218. [CrossRef]

7. Giaquinto, S.; Ring, H. Return to work in selected disabilities. Disabil. Rehabi 2007, 29, 1313-1316. [CrossRef]

8. Morone, G.; Paolucci, S.; Cherubini, A.; De Angelis, D.; Venturiero, V.; Coiro, P.; Iosa, M. Robot-assisted gait training for stroke patients: Current state of the art and perspectives of robotics. Neuropsychiatr. Dis. Treat. 2017, 13, 1303-1311. [CrossRef]

9. Zhang, X.; Yue, Z.; Wang, J. Robotics in Lower-Limb Rehabilitation after Stroke. Behav. Neurol. 2017, 2017, 3731802. [CrossRef]

10. Iosa, M.; Hesse, S.; Oliviero, A.; Paolucci, S. New technologies for stroke rehabilitation. Stroke Res. Treat. 2013, 2013, 815814. [CrossRef] [PubMed]

11. Maceira-Elvira, P.; Popa, T.; Schmid, A.C.; Hummel, F.C. Wearable technology in stroke rehabilitation: Towards improved diagnosis and treatment of upper-limb motor impairment. J. Neuroeng. Rehabil. 2019, 16, 142. [CrossRef] [PubMed]

12. Morone, G.; Spitoni, G.F.; De Bartolo, D.; Ghanbari Ghooschchy, S.; Di Iulio, F.; Paolucci, S.; Zoccolotti, P.; Iosa, M. Rehabilitative devices for a top-down approach. Expert Rev. Med. Devices 2019, 16, 187-195. [CrossRef] [PubMed]

13. Belluscio, V.; Bergamini, E.; Iosa, M.; Tramontano, M.; Morone, G.; Vannozzi, G. The iFST: An instrumented version of the Fukuda Stepping Test for balance assessment. Gait Posture 2018, 60, 203-208. [CrossRef] [PubMed]

14. Belluscio, V.; Bergamini, E.; Tramontano, M.; Orejel Bustos, A.; Allevi, G.; Formisano, R.; Vannozzi, G.; Buzzi, M.G. Gait Quality Assessment in Survivors from Severe Traumatic Brain Injury: An Instrumented Approach Based on Inertial Sensors. Sensors 2019, 19, 5315. [CrossRef] [PubMed]

15. Bergamini, E.; Iosa, M.; Belluscio, V.; Morone, G.; Tramontano, M.; Vannozzi, G. Multisensor assessment of dynamic balance during gait in patients with subacute stroke. J. Biomech. 2017, 61, 208-215. [CrossRef]

16. Tramontano, M.; Bergamini, E.; Iosa, M.; Belluscio, V.; Vannozzi, G.; Morone, G. Vestibular rehabilitation training in patients with subacute stroke: A preliminary randomized controlled trial. NeuroRehabilitation 2018, 43, 247-254. [CrossRef]

17. Raglio, A.; Zaliani, A.; Baiardi, P.; Bossi, D.; Sguazzin, C.; Capodaglio, E.; Imbriani, C.; Gontero, G.; Imbriani, M. Active music therapy approach for stroke patients in the post-acute rehabilitation. Neurol. Sci. 2017, 38, 893-897. [CrossRef]

18. Morone, G.; Tramontano, M.; Iosa, M.; Shofany, J.; Iemma, A.; Musicco, M.; Paolucci, S.; Caltagirone, C. The efficacy of balance training with video game-based therapy in subacute stroke patients: A randomized controlled trial. Biomed. Res. Int. 2014, 2014, 580861. [CrossRef]

19. Lupo, A.; Cinnera, A.M.; Pucello, A.; Iosa, M.; Coiro, P.; Personeni, S.; Gimigliano, F.; Iolascon, G.; Paolucci, S.; Morone, G. Effects on balance skills and patient compliance of biofeedback training with inertial measurement units and exergaming in subacute stroke: A pilot randomized controlled trial. Funct. Neurol. 2018, 33, 131-136.

20. Li, C.; Rusák, Z.; Horváth, I.; Ji, L. Influence of complementing a robotic upper limb rehabilitation system with video games on the engagement of the participants: A study focusing on muscle activities. Int. J. Rehabil. Res. 2014, 37, 334-342. [CrossRef] 
21. Colombo, R.; Raglio, A.; Panigazzi, M.; Mazzone, A.; Bazzini, G.; Imarisio, C.; Molteni, D.; Caltagirone, C.; Imbriani, M. The SonicHand Protocol for Rehabilitation of Hand Motor Function: A validation and feasibility study. IEEE Trans. Neural Syst. Rehabil. Eng. 2019, 27, 664-672. [CrossRef] [PubMed]

22. Mahoney, F.I.; Barthel, D.W. Functional Evaluation: The Barthel Index. Md. State Med. J. 1965, 14, 61-65.

23. Prasad, K.; Kumar, A.; Misra, S.; Yadav, A.K.; Johri, S.; Sarkar, R.S.; Gorthi, S.P.; Hassan, K.M.; Prabhakar, S.; Misra, U.K.; et al. Reliability and validity of telephonic Barthel Index: An experience from multi-centric randomized control study. Acta Neurol. Belg. 2018, 118, 53-59. [CrossRef]

24. Lungenhausen, M.; Lange, S.; Maier, C.; Schaub, C.; Trampisch, H.j.; Endres, H.G. Randomised controlled comparison of the Health Survey Short Form (SF-12) and the Graded Chronic Pain Scale (GCPS) in telephone interviews versus self-administered questionnaires. Are the results equivalent? BMC Med. Res. Methodol. 2007, 7, 50. [CrossRef] [PubMed]

25. Apolone, G.; Mosconi, P.; Quattrociocchi, L. Questionario Sullo Stato di Salute SF-12. Versione Italiana; Guerini e Associati Editore: Milan, Italy, 2005; p. 85. ISBN 8883352017.

26. Galeoto, G.; Colucci, M.; Guarino, D.; Esposito, G.; Cosma, E.; De Santis, R.; Grifoni, G.; Valente, D.; Tofani, M. Exploring Validity, Reliability, and Factor Analysis of the Quebec User Evaluation of Satisfaction with Assistive Technology in an Italian Population: A Cross-Sectional Study. Occup Ther Health Care 2018, 32, 380-392. [CrossRef] [PubMed]

27. Wessels, R.; Persson, J.; Lorentsen, O.; Andrich, R.; Ferrario, M.; Oortwijn, W.; VanBeekum, T.; Brodin, H.; de Witte, L. IPPA: Individually Prioritised Problem Assessment. Technol. Disabil. 2002, 14, 141-145. [CrossRef]

28. Wozniak, M.A.; Kittner, S.J. Return to work after ischemic stroke: A methodological review. Neuroepidemiology 2002, 21, 159-166. [CrossRef]

29. Wang, Y.C.; Kapellusch, J.; Garg, A. Important factors influencing the return to work after stroke. Work 2014, 47, 553-559. [CrossRef]

30. Lindstrom, B.; Roding, J.; Sundelin, G. Positive attitudes and preserved high level of motor performance are important factors for return to work in younger persons after stroke: A national survey. J. Rehabil. Med. 2009, 41,714-718. [CrossRef] [PubMed]

31. Tanaka, H.; Toyonaga, T.; Hashimoto, H. Functional and occupational characteristics predictive of a return to work within 18 months after stroke in Japan: Implications for rehabilitation. Int. Arch. Occup. Environ. Health 2014, 87, 445-453. [CrossRef]

32. Singam, A.; Ytterberg, C.; Tham, K.; von Koch, L. Participation in complex and social everyday activities six years after stroke: Predictors for return to pre-stroke level. PLoS ONE 2015, 10. [CrossRef] [PubMed]

33. Barker-Collo, S.; Feigin, V.L.; Parag, V.; Lawes, C.M.M.; Senior, H. Auckland stroke outcomes study. part 2: Cognition and functional outcomes 5 years poststroke. Neurology 2010, 75, 1608-1616. [CrossRef]

34. Gassert, R.; Dietz, V. Rehabilitation robots for the treatment of sensorimotor deficits: A neurophysiological perspective. J. Neuroeng. Rehabil. 2018, 15, 46. [CrossRef] [PubMed]

35. Masiero, S.; Armani, M.; Rosati, G. Upper-limb robot-assisted therapy in rehabilitation of acute stroke patients: Focused review and results of new randomized controlled trial. J. Rehabil. Res. Dev. 2011, 48, 355-366. [CrossRef] [PubMed]

36. Morone, G.; Masiero, S.; Coiro, P.; De Angelis, D.; Venturiero, V.; Paolucci, S.; Iosa, M. Clinical features of patients who might benefit more from walking robotic training. Restor. Neurol. Neurosci. 2018, 36, $293-299$. [CrossRef] [PubMed]

37. Iosa, M.; Morone, G.; Cherubini, A.; Paolucci, S. The Three Laws of Neurorobotics: A Review on What Neurorehabilitation Robots Should Do for Patients and Clinicians. J. Med. Biol. Eng. 2016, 36, 1-11. [CrossRef] [PubMed]

38. Morone, G.; Bragoni, M.; Iosa, M.; De Angelis, D.; Venturiero, V.; Coiro, P.; Pratesi, L.; Paolucci, S. Who may benefit from robotic-assisted gait training? A randomized clinical trial in patients with subacute stroke. Neurorehabil. Neural Repair 2011, 25, 636-644. [CrossRef] [PubMed]

39. Morone, G.; Iosa, M.; Bragoni, M.; De Angelis, D.; Venturiero, V.; Coiro, P.; Riso, R.; Pratesi, L.; Paolucci, S. Who may have durable benefit from robotic gait training? a 2-year follow-up randomized controlled trial in patients with subacute stroke. Stroke 2012, 43, 1140-1142. [CrossRef] [PubMed] 
40. Paolucci, S.; Iosa, M.; Toni, D.; Barbanti, P.; Bovi, P.; Cavallini, A.; Candeloro, E.; Mancini, A.; Mancuso, M.; Monaco, S.; et al. Neuropathic pain special interest group of the Italian Neurological Society. Prevalence and Time Course of Post-Stroke Pain: A Multicenter Prospective Hospital-Based Study. Pain Med. 2016, 17, 924-930. [CrossRef]

41. Iosa, M.; Lupo, A.; Morone, G.; Baricich, A.; Picelli, A.; Panza, G.; Smania, N.; Cisari, C.; Sandrini, G.; Paolucci, S. Post Soft Care: Italian implementation of a post-stroke checklist software for primary care and identification of unmet needs in community-dwelling patients. Neurol. Sci. 2018, 39, 135-139. [CrossRef]

42. Han, J.; Lee, H.I.; Shin, Y.-I.; Son, J.H.; Kim, S.-Y.; Kim, D.Y.; Sohn, M.K.; Lee, J.; Lee, S.-G.; Oh, G.-J.; et al. Factors influencing return to work after stroke: The Korean Stroke Cohort for Functioning and Rehabilitation (KOSCO) Study. BMJ Open 2019, 9, 11. [CrossRef] [PubMed]

43. Bragoni, M.; Broccoli, M.; Iosa, M.; Morone, G.; De Angelis, D.; Venturiero, V.; Coiro, P.; Pratesi, L.; Mezzetti, G.; Fusco, A.; et al. Influence of psychologic features on rehabilitation outcomes in patients with subacute stroke trained with robotic-aided walking therapy. Am. J. Phys. Med. Rehabil. 2013, 92, e16-e25. [CrossRef] [PubMed]

(C) 2020 by the authors. Licensee MDPI, Basel, Switzerland. This article is an open access article distributed under the terms and conditions of the Creative Commons Attribution (CC BY) license (http://creativecommons.org/licenses/by/4.0/). 